\title{
A chimeric platelet-targeted urokinase prodrug selectively blocks new thrombus formation
}

\author{
Rudy E. Fuentes, ${ }^{1}$ Sergei Zaitsev, ${ }^{2,3}$ Hyun Sook Ahn, ${ }^{1}$ Vincent Hayes, ${ }^{1}$ M. Anna Kowalska, ${ }^{1}$ Michele P. Lambert, ${ }^{4}$ Yuhuan Wang, ${ }^{1}$ \\ Donald L. Siegel, ${ }^{5}$ Daniel W. Bougie, ${ }^{6}$ Richard H. Aster, ${ }^{6}$ Daniel D. Myers, ${ }^{7}$ Victoria Stepanova, ${ }^{5}$ Douglas B. Cines, ${ }^{5}$ \\ Vladimir R. Muzykantov, ${ }^{2,3}$ and Mortimer Poncz ${ }^{1,4}$
}

'Division of Hematology, The Children's Hospital of Philadelphia (CHOP), Philadelphia, Pennsylvania, USA. Institute for Translational Medicine and Therapeutics, ${ }^{3}$ Department of Pharmacology, ${ }^{4}$ Department of Pediatrics, and ${ }^{5}$ Department of Pathology and Laboratory Medicine, Perelman School of Medicine, University of Pennsylvania, Philadelphia, Pennsylvania, USA. ${ }^{6}$ Blood Research Institute, Blood Center of Wisconsin, Milwaukee, Wisconsin, USA. Department of Surgery, University of Michigan Health Systems, Ann Arbor, Michigan, USA.

\begin{abstract}
The use of fibrinolytic agents to prevent new thrombus formation is limited by an increased risk of bleeding due to lysis of hemostatic clots that prevent hemorrhage in damaged blood vessels. We sought to develop an agent that provides thromboprophylaxis without carrying a significant risk of causing systemic fibrinolysis or disrupting hemostatic clots. We previously showed that platelet (PLT) $\alpha$ granule-delivered urokinase plasminogen activator (UPA) is highly effective in preventing thrombosis, while being associated with little systemic fibrinolysis or bleeding. Here, we generated a chimeric prodrug composed of a single-chain version of the variable region of an anti- $\alpha$ llb $\beta 3 \mathrm{mAb}$ fused to a thrombin-activatable, low-molecular-weight pro-uPA (PLT/uPA-T). PLT/uPA-T recognizes human $\alpha$ llb $\beta 3$ on both quiescent and activated platelets and is enzymatically activated specifically by thrombin. We found that this prodrug binds tightly to human platelets even after gel filtration, has a prolonged half-life in mice transgenic for human allb compared with that of uPA-T, and prevents clot formation in a microfluidic system. Importantly, in two murine injury models, PLT/uPA-T did not lyse preexisting clots, even when administration was delayed by as little as 10 minutes, while it concurrently prevented the development of nascent thrombi. Thus, PLT/uPA-T represents the prototype of a platelet-targeted thromboprophylactic agent that selectively targets nascent over preexisting thrombi.
\end{abstract}

\section{Introduction}

There is a substantial and important unmet need to prevent thrombus formation without lysing preexisting hemostatic clots, especially in settings in which the risks of both thrombosis and bleeding are high. (1) This situation occurs commonly, for example, after major general or orthopedic surgery, in which the risk of thrombosis in the absence of effective intervention may exceed $50 \%$, and mortality from symptomatic pulmonary embolism approaches $10 \%$ (2). Yet, the risk of perioperative bleeding often delays the introduction of anticoagulation by several days. Similar tradeoffs in the timing of anticoagulation based on the risk of bleeding arise in patients with ischemic stroke and in those who have sustained traumatic brain injury or extensive general trauma. In these and related settings, there is a clear need for therapies that distinguish between preexisting hemostatic clots and nascent, potentially occlusive thrombi.

Fibrinolytic agents such as tissue-type plasminogen activator (tPA) and urokinase plasminogen activator (uPA) are highly effective at lysing thrombi, but have short half-lives in the circulation, cause systemic fibrinolysis, and predispose patients to

Authorship note: R.E. Fuentes, S. Zaitsev, and H. Sook Ahn contributed equally to this work.

Conflict of interest: The authors have declared that no conflict of interest exists. Submitted: February 12, 2015; Accepted: November 12, 2015.

Reference information: / Clin Invest. 2016;126(2):483-494. doi:10.1172/JCI81470. bleeding, because they permeate preexisting hemostatic clots (3). Moreover, both PAs have vasoactive and neurotoxic side effects $(4,5)$ due to signaling through identified cell-surface receptors (5) and diffusion through the blood-brain barrier after stroke. Therefore, the clinical application of fibrinolytic agents has been limited primarily to the management of a small subset of patients with acute ischemic stroke or acute life- or limb-threatening thromboembolism $(6,7)$.

One potential way to rectify the short in vivo lifespan, nonselective permeation of hemostatic and vaso-occulsive clots, deleterious interaction with cellular receptors, and permeation into neurologic and other tissues is to harness the drug to cells that reside within the blood. We have used this approach successfully by linking PAs to rbc and shown that this approach provides enduring and safe thromboprophylaxis in several animal models (8). However, rbc are not rapidly and selectively recruited to sites of incipient thrombus formation, unlike platelets, which provide procoagulant surfaces in a temporally and regionally restricted manner. This raises the possibility that targeting PAs to the surface of platelets would enhance their delivery and invert the function of platelets from prothrombotic to fibrinolytic and do so in a manner that would not lyse mature clots that are no longer recruiting platelets.

In support of this possibility, it is known that Quebec platelet disorder (QPD), a mild-to-moderate inherited platelet disorder, is caused by the ectopic expression and storage of UPA in platelet $\alpha$ granules (9). Bleeding in these patients is typically restricted 
to surgical stress and is responsive to inhibitors of PAs such as tranexamic acid (TEA) (10). A transgenic mouse expressing uPA in the $\alpha$ granules of circulating platelets has a phenotype similar to that of QPD patients (11). These QPD mice do not bleed spontaneously or develop systemic fibrinolysis, but they are capable of preventing the formation of occlusive thrombi in a $\mathrm{FeCl}_{3}$ carotid injury model and a pulmonary microembolism model (11). Importantly, it is only necessary to infuse uPA-expressing platelets into WT mice to a level of approximately $5 \%$ of total platelets to completely prevent occlusive thrombi in the $\mathrm{FeCl}_{3}$ injury model without causing noticeable bleeding (11). These studies suggest that the release of activated PAs within growing thrombi is especially effective in preventing further expansion and may help explain why over $90 \%$ of the PA inhibitor 1 (PAI-1) available in the circulation is stored in platelet $\alpha$ granules (12).

To test this hypothesis and to translate it into a clinically viable thromboprophylactic agent, we generated a construct that has an N-terminal single-chain variable fragment ( $\mathrm{scFv}$ ) of a $\mathrm{mAb}$ directed against human $\alpha \mathrm{IIb}$ (CD41), which is part of the most abundant receptor on the platelet surface (13), fused to a low-molecular-weight (LMW) pro-uPA (thrombin-activatable, LMW pro-uPA [PLT/uPA-T]) into which a thrombin-specific cleavage and activation site was introduced at its N-terminus (Figure 1A). Here, we characterize platelet targeting of this prodrug and demonstrate its thrombolytic efficacy both in vitro and in vivo. We established what we believe to be two novel dualinjury murine models that show that PLT/uPA-T selectively prevents the generation of occlusive nascent thrombi, relatively sparing even recently formed clots from lysis (Figure 1B). The clinical implications of these findings are discussed herein.

\section{Results}

Generation and in vitro characterization of PLT/UPA-T. Our goal was to develop a thrombin-activatable thrombolytic prodrug that would target the surface of human platelets without perturbing their function. The targeted platelets would then serve as natural carriers to localize the prodrug in nascent thrombi, which are actively incorporating platelets and where the prodrug could be activated by thrombin (Figure 1B). We modeled our platelettargeting construct on a previously described rbc-targeting scFv/ uPA-T (8). In PLT/uPA-T, the rbc scFv is replaced with a PLT scFv derived from an anti-human $\alpha \mathrm{IIb} \mathrm{mAb}$ at its N-terminus (Figure $1, C$ and D). We selected the $\alpha \operatorname{IIb} \beta 3$ receptor as the plateletspecific vascular target in part because of its abundance on the platelet surface, which would allow for a wide dosing range depending on the indication (13). A series of anti-human $\alpha \mathrm{IIb}-$ specific mAbs were tested. The mAb 312.8 was selected on the basis of its high affinity for human platelets, lack of binding to WT mouse platelets, binding to h $\alpha \mathrm{IIb}^{+}$mouse platelets (data not shown and Supplemental Figure 1A; supplemental material available online with this article; doi:10.1172/JCI81470DS1), and lack of interference with platelet aggregation (Supplemental Figure 1B).

PLT/uPA-T contained the same LMW uPA zymogen (uPA-T) on its $\mathrm{C}$-terminus as the rbc fusion protein and consisted of a deletion of two amino acids that lead to loss of a plasmin cleavage site and creation of a thrombin activation site that must be proteolyzed before fibrinolytic activity is expressed in vitro (Figure $2 \mathrm{~A}$ ). $\mathrm{uPA}-\mathrm{T}$ and PLT/uPA-T are inactivated by the lysine analog TEA, which blocks binding of plasminogen to fibrin (14). Kinetics studies showed that the rate of activation of PLT/uPA-T by thrombin was almost 2 times slower than that of uPA-T (Supplemental Figure 2A), though thrombin cleavage of both appeared sensitive to the presence of antithrombin (Supplemental Figure 2B). Using a series of serine proteases, the proteolytic activation of PLT/uPA-T was found to be restricted to thrombin (Figure 2B).

Binding of PLT/uPA-T to nonactivated and phorbol myristate acetate-activated (PMA-activated) human platelets was identical as assessed by flow cytometry (Figure $3 \mathrm{~A}$ ). In contrast, PLT/uPA-T did not bind to quiescent or activated WT murine platelets but did bind to both states of haIIb+ murine platelets, which express human $\alpha$ IIb complexed to mouse $\beta 3$ (15) (Figure 3A). PLT/uPA-T bound immobilized human $\alpha \operatorname{IIb} \beta 3$ with a $K_{D}$ of approximately 5 $\mathrm{nM}$ (Figure 3B). PLT/uPA-T did not interfere with aggregation of human platelets by adenosine diphosphate (ADP) (Figure 3C).

Microfluidic thrombosis studies using gel-filtered platelets. To test platelet delivery of PLT/uPA-T and to begin to determine fibrinolytic efficacy, isolated human platelets were incubated with various concentrations of PLT/uPA-T or UPA-T prodrug and then gel filtered (Figure 4A). The filtered platelets were then added back to blood to reconstitute "whole blood," and clotting was activated by either adding factor VIIa (FVIIa) (16) or by producing a heparininduced thrombocytopenia-like (HIT-like) prothrombotic state by adding PF4 and the HIT-like mAb KKO (17). The samples were then flowed into a microfluidic channel (Figure 4A). PLT/uPA-T, but not uPA-T, was detected on the platelet surface after gel filtration (Figure 3A). Compared with uPA-T at a comparable starting dose, PLT/uPA-T significantly decreased thrombus development, as indicated by a reduction in both fibrin and platelet accumulation after addition of FVIIa (Figure 4, B and C, respectively) or after induction of an HIT-like state (Supplemental Figure 3, A and B). Similar studies were performed using baboon platelets, which also bound PLT/uPA-T (Supplemental Figure 4A). Again, PLT/ uPA-T blocked thrombus growth, as indicated by decreased fibrin and platelet accumulation in the microfluidic system relative to uPA-T (Supplemental Figure 4, B and C, respectively). These data are consistent with our finding that PLT/UPA-T bound to the platelet surface remains present after gel filtration, while uPA-T does not bind to the platelet surface and is lost with filtration.

Thrombolytic prodrug half-life and systemic effects in mice. Labeled uPA-T and PLT/uPA-T were infused into WT and hoIIb mice, and binding to the platelet surface was monitored. PLT/ uPA-T bound to haIIb ${ }^{+}$with a half-life of approximately 2 hours, and bound drug was still detectable at 24 hours (Figure 5, A and B). In contrast, PLT/uPA-T did not bind to WT platelets at any concentration tested (data not shown), and UPA-T did not bind to any source of platelets (Figure 5A and data not shown for WT mice studies). Intravenous infusion of PLT/uPA-T or UPA-T did not cause a significant fall in platelet numbers compared with that seen in the PBS control (Figure 5C). A bolus of either uPA-T or PLT/uPA-T followed by infusion did not cause a rise in D-dimers or a significant fall in fibrinogen levels (Figure 5D).

Models of nascent versus preexisting thrombi: $\mathrm{FeCl}_{3}$ carotid arterial versus tail-clipping injuries. The primary goal in developing PLT/uPA-T was to be able to selectively lyse nascent pathological 

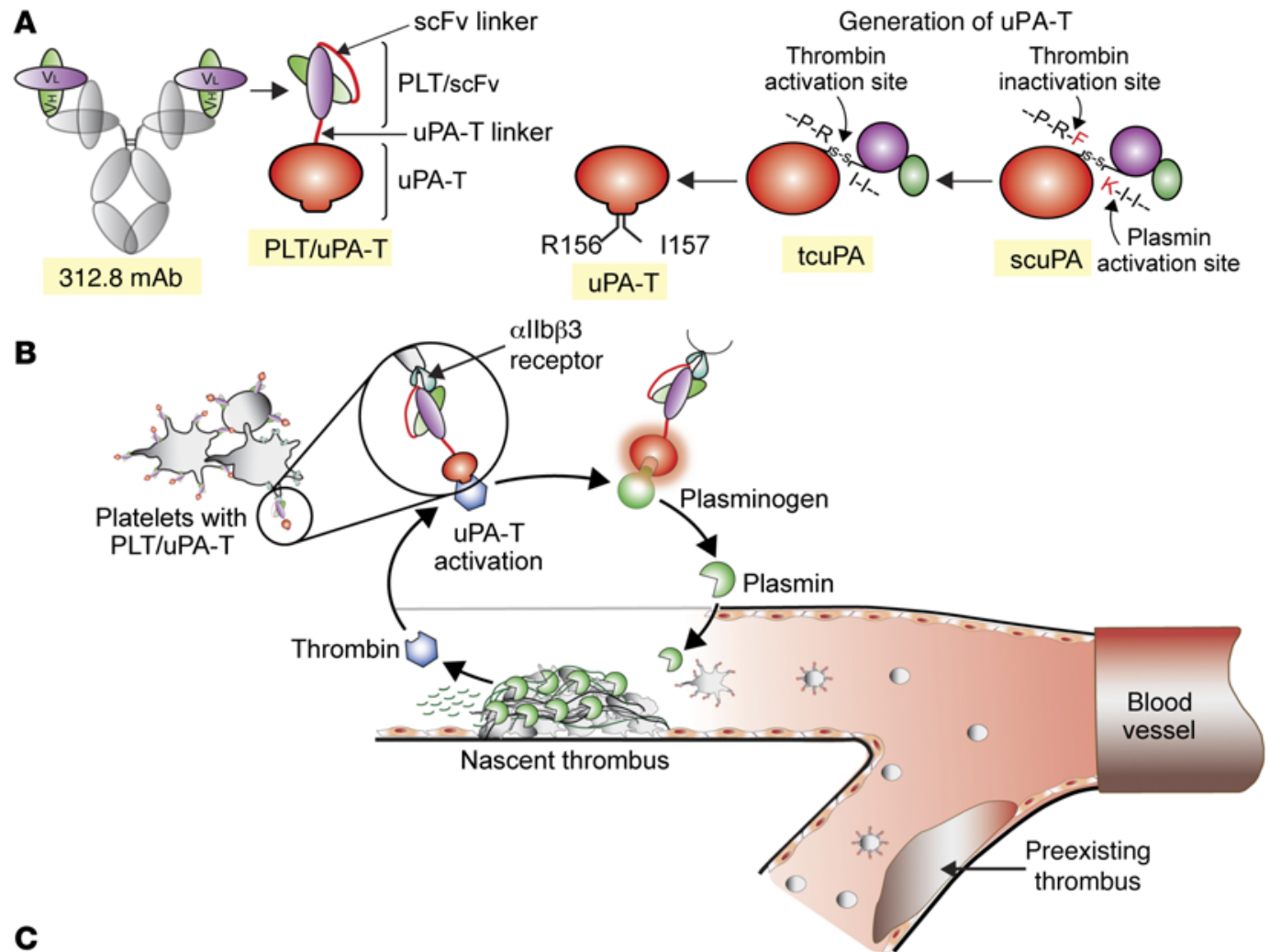

Ncol

CCATGGAAGGTGAAGCTGGTGGAGTCAGGGGGGGCTTAGTGAGGCCTGGAGGGTCCCTGAAACTCTCCTGTGCAGCCTCTGGATTCACTTTCAGTAGCTATACCATGTCTTGGGTTCGCCAC ACTCCGGAGAaGAGGCTGGAGTGGGTCGCAACCCTTAGTAGTGTTGGTAGTTACACCTACTATGCAGGCAGTGTGAaGGGCCGATTCACCATCTCCAGAGACAATGCCAAGAaCACCCTGCAC

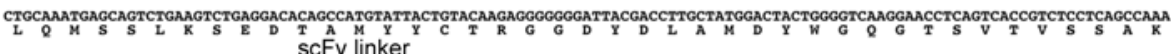
ScFv linker

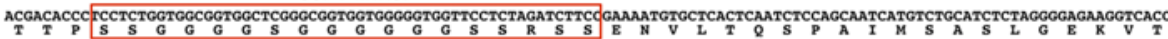

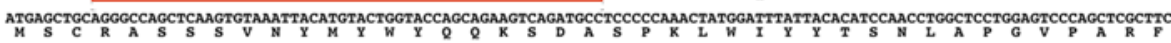

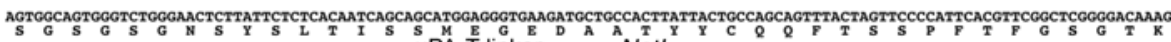
(5)

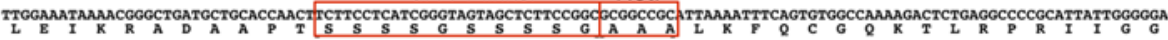

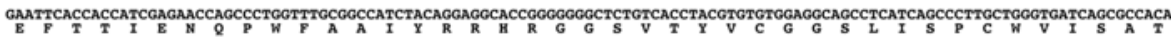

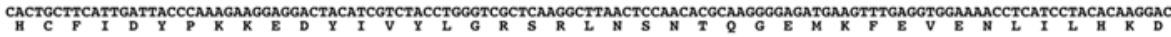

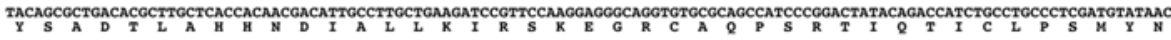

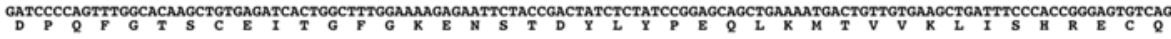

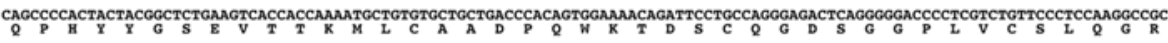

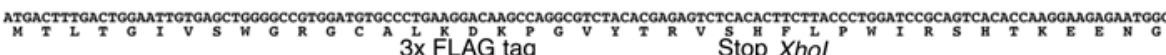
crgocccrce

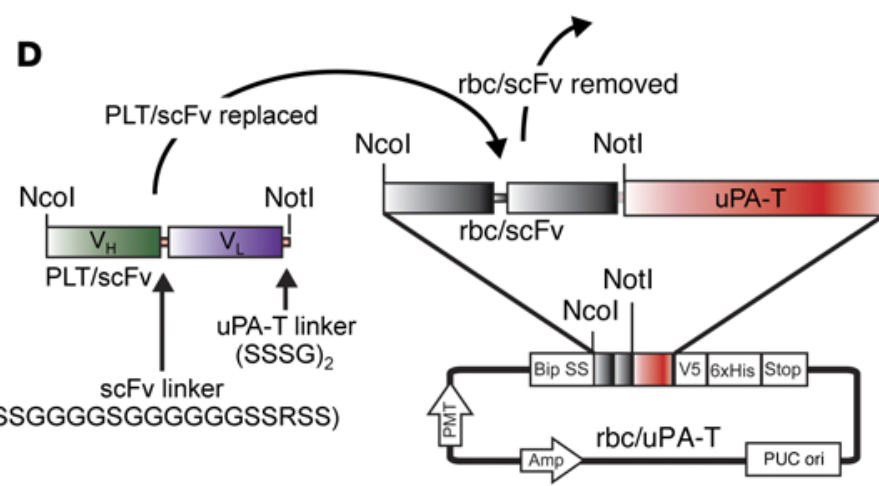

Figure 1. Schematics of the construction and biology of PLT/uPA-T. (A) Schematic construct of the scFv N-terminal and LMW UPA-T C-terminal portions of PLT/ uPA-T. Right: C-terminal zymogen of human single-chain uPA (scuPA) lacking the full molecule domains and modified by deleting Phe157 and Lys158 (in red), which convert a plasmin activation site into the thrombin-activatable uPA-T. tcuPA, two-chain urokinase; $P$, proline; $R$, argine; $F$, phenylalanine; $K$, lysine; I, isoleucine; s-s, cysteine disulfide bonds. (B) Intended biology of a chimeric PLT/UPA-T that would bind to the surface of resting or activated platelets without activating these cells and be attracted to a nascent thrombus, whereby available thrombin would activate the urokinase moiety uPA-T, leading to plasminogen activation and subsequent fibrinolysis. Preexisting clots, which do not bind new platelets except transiently within the shell and have little or no active thrombin, or at least no accessible thrombin, are protected from Iysis by PLT/UPA-T. (C) Schematic of the final expression vector for PLT/UPA-T in insect cells with removal of the rbc/scFv. Bip SS, Ig-binding protein; V5, simian virus 5 epitope; 6 XHis, polyhistidine peptide; PMT, metallothionein promoter; Amp, ampicillin-resistant gene; PUC ori, plasmid origin of replication. (D) DNA sequence of the PLT/UPA-T insert in C, with key components boxed in red. 
A

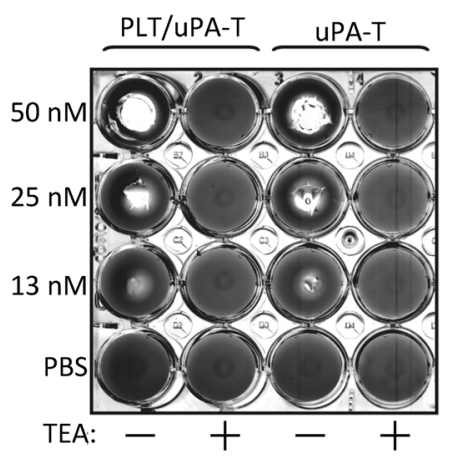

B

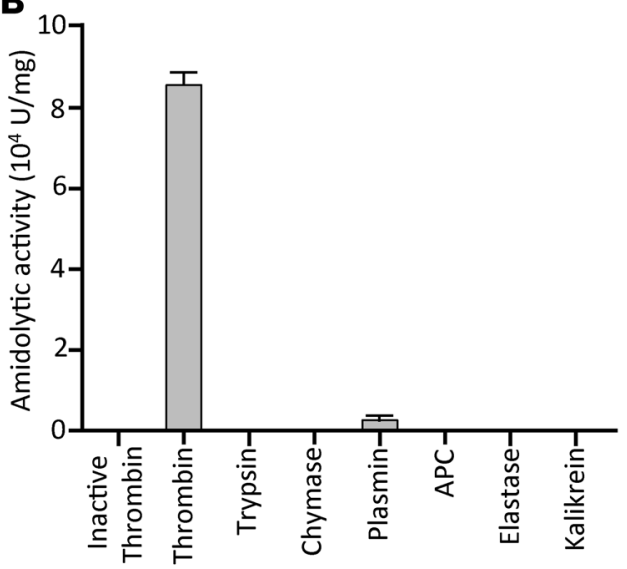

Figure 2. Fibrinolytic characterization of PLT/UPA-T. (A) In vitro fibrinolysis of equimolar amounts of UPA-T and PLT/UPA-T in the presence of thrombin $(20 \mathrm{nM})$ rendered them equally active, as evidenced by the size of lytic zones in a fibrin plate analysis. This fibrinolysis could be blocked by TEA $(1 \mu \mathrm{g} / \mathrm{ml})$. (B) Exposure of PLT/UPA-T to various proteases confirmed activation of PLT/UPA-T solely by thrombin. Data represent the mean \pm 1 SEM. $n=3$ experiments performed in duplicate. thrombi without affecting preexisting clots that might be required for hemostasis. Several thrombosis models were used to determine whether PLT/uPA-T was capable of demonstrating those abilities. In the first model, haIIb ${ }^{+}$mice were studied to examine the ability of the prodrug to prevent nascent thrombus formation after a $\mathrm{FeCl}_{3}$-sclerosing carotid arterial injury, while sparing loss of hemostatic stability in preexisting tail-clip injuries (Figure 6, A and B). Prodrug dosing was based on the studies in Figure 4 and Supplemental Figure 3 and 4. Prodrugs were injected as a bolus, followed by continuous infusion of the same dose over the ensuing 30 minutes (bolus infusion). We chose this approach because a bolus of $5 \mathrm{mg} / \mathrm{kg}$ uPA-T - in contrast to a bolus of $0.5 \mathrm{mg} / \mathrm{kg}$ PLT/uPA-T - was ineffective in preventing thrombus formation in the $\mathrm{FeCl}_{3}$ carotid artery injury model, consistent with the short half-life of uPA-T (Figure 6A). In contrast, using a bolus of uPA-T, followed by a 30-minute infusion of $5 \mathrm{mg} / \mathrm{kg}$ uPA-T, was as effective as 0.5 or $2.5 \mathrm{mg} / \mathrm{kg}$ PLT/uPA-T in preventing nascent thrombi from forming (Figure 6A). At a bolus infusion dose of 5 $\mathrm{mg} / \mathrm{kg}$ uPA-T, an increase in rebleeding was observed in the tailclipping studies, while no increase was seen with lower $\mathrm{mg} / \mathrm{kg}$ uPA-T doses or with any tested dose of PLT/uPA-T (Figure 6B and Supplemental Figure 5).

We sought to examine the biology of PLT/uPA-T in the presence of human platelets in vivo. To do so, we studied the effects of PLT/uPA-T, uPA-T, and uPA alone in NSG mice infused with human platelets to a target level of $20 \%$ of total circulating platelets. At doses of UPA or UPA-T that prevented nascent clots from forming in the $\mathrm{FeCl}_{3}$ model (Figure 6C), loss of hemostatic integrity was seen in the preexisting tail-clipping model (Figure 6D and Supplemental Figure 5). In contrast, at an effective dose of PLT/uPA-T (Figure 6C), little loss of hemostatic integrity was seen (Figure 6D). Moreover, unlike uPA and uPA-T, thromboprophylaxis with PLT/uPA-T was dependent on the presence of human platelets (Figure 6C). Thus, PLT/uPA-T was delivered to the site of injury by the human platelets. Without the presence of human platelets, PLT/uPA-T lost its effectiveness as a thromboprophylactic agent.

Models of nascent versus preexisting thrombi: cremaster arteriole laser injuries. Our tentative conclusion that PLT/uPA-T effectively lyses nascent, but not preexisting, clots could have resulted from our use of two distinct injury models to study nascent ver- sus established clots. To address this concern, we next used a single-injury model involving laser-induced cremaster arteriolar injury in xenotransfused NSG mice to study thromboprophylactic efficacy in the setting of human platelets (Figure 7A). A series of laser injuries was induced after a bolus infusion of prodrug to create nascent thrombi. As expected, PBS infusion had no effect on the incorporation of fibrin or platelets into these clots (Figure 7B and Supplemental Videos 1 and 2), but a 5-mg/ $\mathrm{kg}$ bolus infusion dose of either uPA or uPA-T doses was equally as effective as a $0.15-\mathrm{mg} / \mathrm{kg}$ dose of PLT/uPA-T in preventing fibrin and platelet incorporation into nascent thrombi (Figure 7B, Supplemental Videos 3 and 4 [uPA], and Supplemental Videos 5 and 6 [PLT/ uPA-T]). Thus, in this model, PLT/uPA-T appeared to be approximately 30 -fold more effective than UPA or UPA-T. These bolus infusion doses, however, had different effects on preexisting clots. Compared with a PBS bolus infusion, uPA or UPA-T bolus infusion markedly decreased the size of preexisting clots, while bolus infusion of PLT/uPA-T was closer to not having included a fibrinolytic agent (Figure 7C and Supplemental Videos 7-10 [PBS] versus Supplemental Videos 11-14 [uPA] versus Supplemental Videos 15-18 [PLT/uPA-T]).

\section{Discussion}

PLT/uPA-T provides a unique combination of features that target a potent fibrinolytic prodrug to nascent clots, while exerting limited thrombolysis of even recently established clots. First, binding to circulating platelets prolongs the prodrug's lifespan and facilitates its delivery to nascent clots due to natural platelet tropism. Second, platelet carriage inhibits the penetration of existing clots by soluble proenzyme uPA-T, thereby attenuating hemorrhage and probably other adverse effects in surrounding tissues. Third, $\mathrm{uPA}-\mathrm{T}$ is a prodrug that is not subject to rapid inactivation and clearance by PAI-1. Fourth, PLT/uPA-T is activated by thrombin and not by the other serine proteases tested, including plasmin (8). Thrombin is preferentially expressed on the shell of nascent thrombi, where platelet turnover is occurring, whereas in preexisting clots, any residual thrombin is sequestered within the core, where it is less accessible to platelets (18). Drugs used in previous attempts to target uPA to platelets have lacked the thrombin activation mechanism, had short circulating half-lives, and failed to avoid systemic fibrinolysis (19). 
A

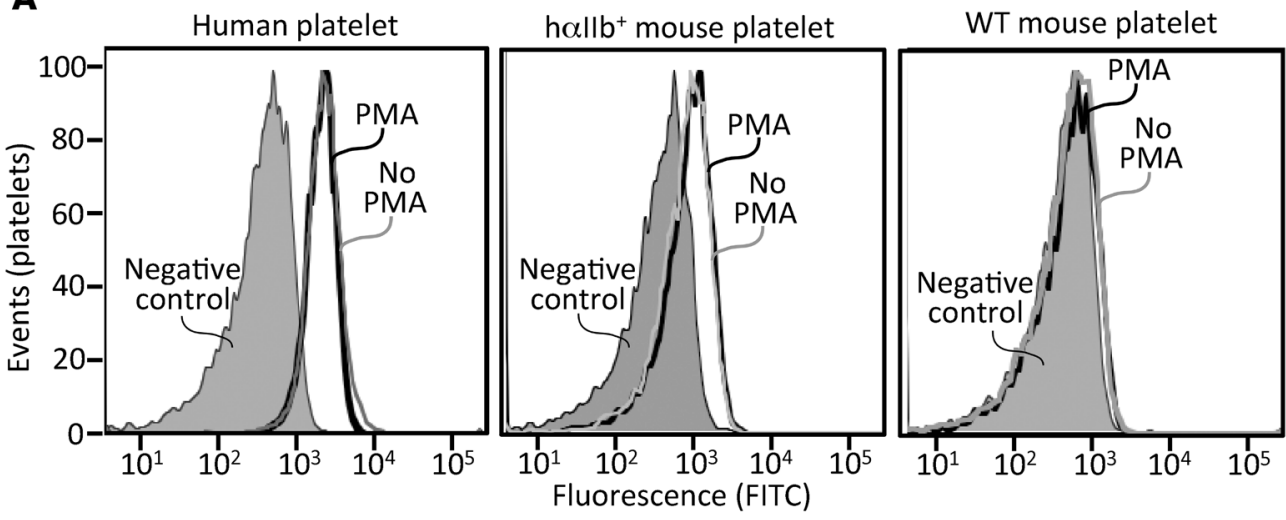

Figure 3. Platelet-binding properties of PLT/uPA-T. (A) Binding of PLT/UPA-T to nonactivated and PMA-activated human (left), hall $b^{+}$mouse (middle), and WT mouse (right) platelets. (B) Binding of ${ }^{125}$ I-PLT/UPA-T to human $\alpha$ llb $\beta 3$ or BSA (nonspecific binding) by ELISA. (C) Human platelet aggregation with the ADP agonist $(10 \mu \mathrm{M})$ in the absence or presence of PLT/uPA-T $(30 \mu \mathrm{g} / \mathrm{ml})$.

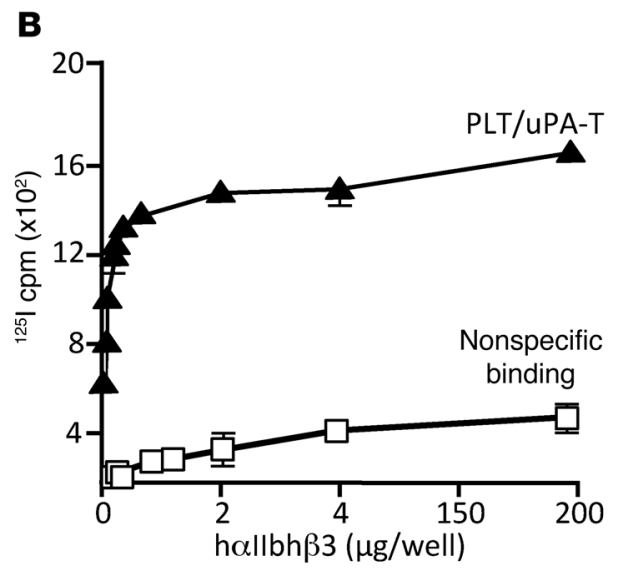

C

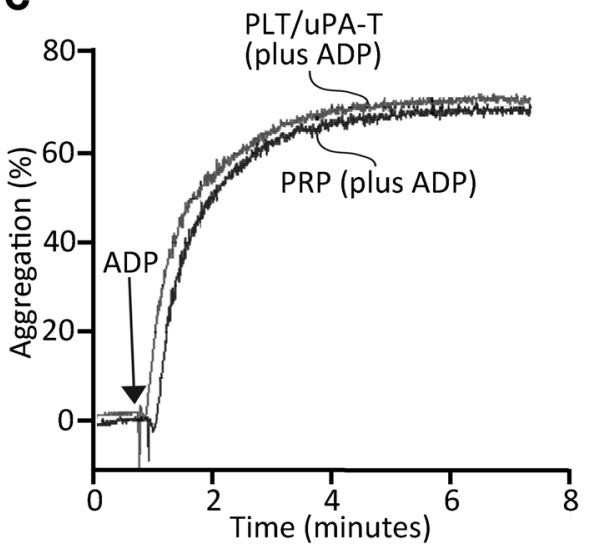

PLT/uPA-T binds to both quiescent and activated human (and baboon) platelets in vitro and in vivo and neither induces nor inhibits platelet activation, thereby maintaining normal hemostatic pathways that drive platelets into clots. When injected into $\mathrm{h} \alpha \mathrm{IIb}^{+}$mice that expressed $\mathrm{h} \alpha \mathrm{IIb} / \mathrm{m} \beta 3$ on their platelets (15), the half-life of PLT/UPA-T was approximately 2 hours (Figure $5 \mathrm{~A}$ ), which is approximately 100 -fold longer than that of nontargeted uPA (20). This combination of high affinity for platelets and a prolonged half-life of PLT/uPA-T translated into an approximately 30 -fold greater inhibition of clot growth than was seen with uPA-T alone using a bolus infusion approach (Figure 7), and an even greater difference if only a bolus was given (Figure 6). This difference was observed in spite of PLT/ uPA-T being activated 2 times less efficiently by thrombin than was seen with uPA-T (Supplemental Figure 2). To circumvent the shorter half-lives of uPA and uPA-T, in vivo comparative studies were performed using a bolus infusion format. This intervention paradigm established that the increased thrombolytic activity of PLT/uPA-T was not solely due to its longer half-life, but was driven by platelet delivery to the clot.

Importantly, PLT/uPA-T, but not UPA or uPA-T, selectively lysed nascent versus preexisting clots when comparing both carotid arterial thrombosis with tail-clip rebleeding and a laser injury to cremaster arterioles before and after drug delivery. In both formats, the efficacy of PLT/uPA-T depended on the presence of human platelets for binding and delivery. The common injury model affirms that the selective agnosia of PLT/uPA-T for preformed clots is not the result of differences in sensitiv- ity between models (e.g., carotid artery vs. tail-clip models), but can be seen when using a single model for the nascent and preexisting clots (e.g., before or after injuries in the cremaster arteriole laser injury model).

The importance of platelet targeting by the PLT/uPA-T is supported by our NSG mouse studies (Figures 6 and 7) showing that the fibrinolytic effects of PLT/uPA-T were only seen if the animals had been transfused with human platelets. This contrasted with UPA and UPA-T, whose fibrinolytic effects were seen with or without infused human platelets. Moreover, PLT/uPA-T lysed nascent clots as effectively as did the 30-fold-higher doses of uPA or UPA-T, even when less than $20 \%$ of the platelets were of human origin. These results are reminiscent of previous studies showing that thrombosis is prevented in WT mice when their platelet composition contains as little as $5 \%$ transfused QPD platelets (11) and affirm the potency of platelets as carriers of thrombolytic agents, and probably other cargos as well, to nascent clots.

In designing PLT/uPA-T as the target for the scFv moiety, we chose $\alpha \operatorname{IIb} \beta 3$, which is the most prevalent receptor on the surface of platelets, with up to 80,000 copies per platelet after activation (13). We selected an $\mathrm{Ab}$ template for the scFv part of the fusion that did not inhibit platelet aggregation (Figure 3C) and did not distinguish nonactivated from activated $\alpha \operatorname{IIb} \beta 3$. This enabled us to target all platelets, extend the drug's half-life, and not perturb the normal targeting of platelets to sites where thrombi were forming. A previously reported chimeric construct of CD39 and also uPA with an $\alpha \operatorname{IIb} \beta 3 \mathrm{scFv}$ chimera $(21,22)$ bound only to activated $\alpha \operatorname{IIb} \beta 3$. The circulating half-life of this $\mathrm{scFv} / \mathrm{CD} 39$ chimera 
A

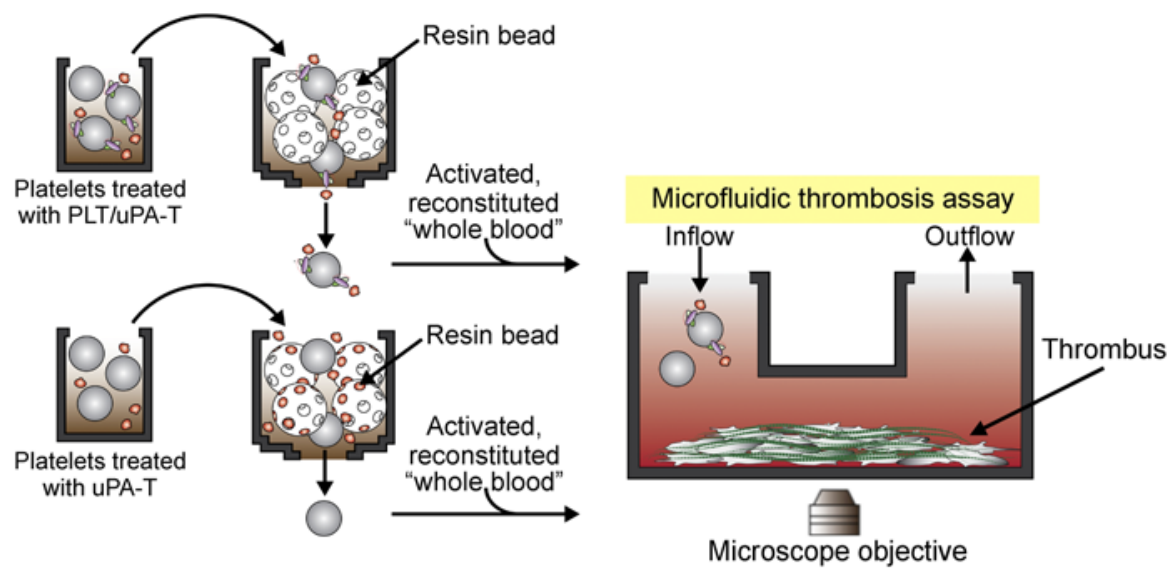

B

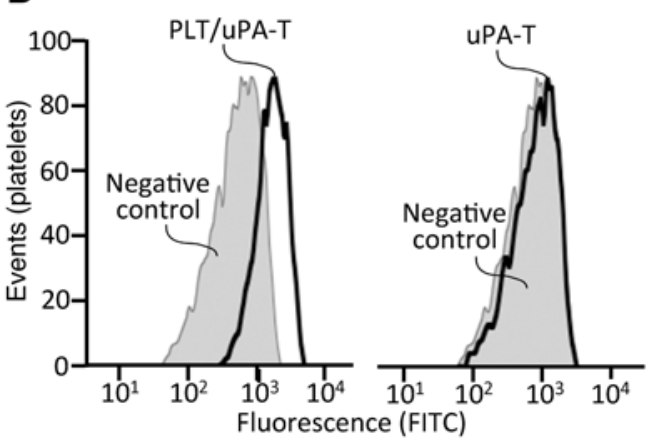

C

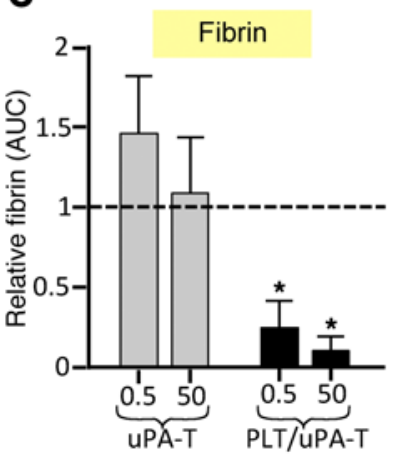

D

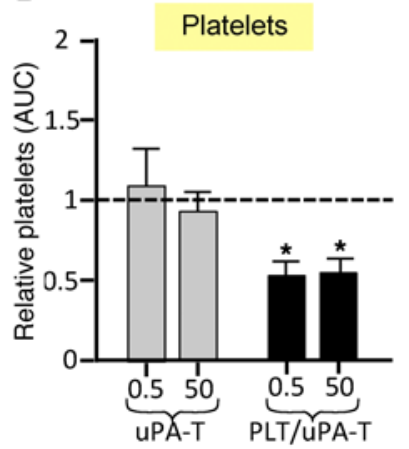

Figure 4. Thrombolytic effects of PLT/uPA-T in human blood. (A) Schematic beginning with isolated human platelets incubated with prodrugs or control PBS and then gel filtered. These platelets are then used to reconstitute whole blood. Clotting in whole blood is activated by adding either FVIla or PF4 and the HIT-like mAb KKO to induce a prothrombotic state. The blood is then flowed through a vWF-coated channel, and adherence of platelets and fibrinogen to the channel's wall is monitored. (B) The prodrugs uPA-T $(10 \mu \mathrm{g} / \mathrm{ml})$ and PLT/uPA-T $(10 \mu \mathrm{g} / \mathrm{ml})$ were incubated with human platelets and then gel filtered. Representative flow cytometric studies of platelet surface-bound prodrug after gel filtration are shown. (C) Relative fibrin accumulation and (D) platelet deposition in the microfluidic channel of reconstituted "whole" blood activated by FVIla with platelet-bound prodrug is shown compared with preincubation with PBS (dashed line). Data represent the mean \pm 1 SEM. $n=5$ independent experiments, each performed in duplicate. ${ }^{*} P \leq 0.001$ compared with PBS. Data analysis was done by Student's $t$ test for drug-treated samples compared with PBS controls.

was not reported, but may have been very short, as it would have few, if any, receptors in the circulation, and its uPA portion would not be protected from proteolysis. On the other hand, the scFv/ CD39, and perhaps the scFv/uPA, had a longer than 2-minute half-life on incorporated platelets within the thrombi, which presumably expressed activated surface $\alpha \operatorname{IIb} \beta 3$. This helps to explain the observed lack of an effect from the $\mathrm{scFv}$ chimera proteins when given as a bolus 3 minutes before a tail-clip injury, a setting without activated platelets to which these drugs can attach and prolong their half-lives. Presumably, in this setting, the drugs are cleared rapidly, and the tail-clip assay is unaffected. In contrast, when these drugs are given 3 minutes after the initiation of an $\mathrm{FeCl}_{3}$ carotid arterial injury, activated platelets are likely to have attached to the site of injury, bind the scFv chimeras, and initiate lysis (21). Therefore, we believe that these chimeras need to be tested using a bolus infusion format to eliminate these pharmacokinetic concerns and to better simulate the clinical usage of these chimeric drugs. These concerns also underscore the notion that the biology and clinical utility of platelet-directed drugs may be significantly influenced by the platelet receptor targeted, that this binding requires that receptor to be in a specific state of acti- vation, and that the activated drug component of the chimera is subject to inactivation in the circulation.

We envision PLT/uPA-T being especially useful in settings such as after major pulmonary embolism, stroke, or trauma or following extensive surgery in which the risks of thrombosis are high, as is the possibility of hemorrhage due to breakdown of preexisting clots. Currently, thromboprophylaxis is routinely delayed for hours to days in these common situations because of the real concern for bleeding. Whether use of PLT/uPA-T, which exhibits a lead time as short as 10 minutes to seal and protect clots in mouse models, will prove applicable to human hemostasis will, of course, require additional studies. Studies will also be needed to determine whether this strategy will be useful in systemic prothrombotic states, including heparin-induced thrombocytopenia (Figure 3 and Supplemental Figure 4), in which the intent is to prevent disease progression by lysing nascent thrombi and preventing the onset of major vessel occlusion.

In summary, we have designed what we believe to be a novel PLT/uPA-T prodrug that selectively lyses nascent thrombi with relative sparing of preexisting clots. This new agent combines several important features: (a) the prodrug requires thrombin 
A

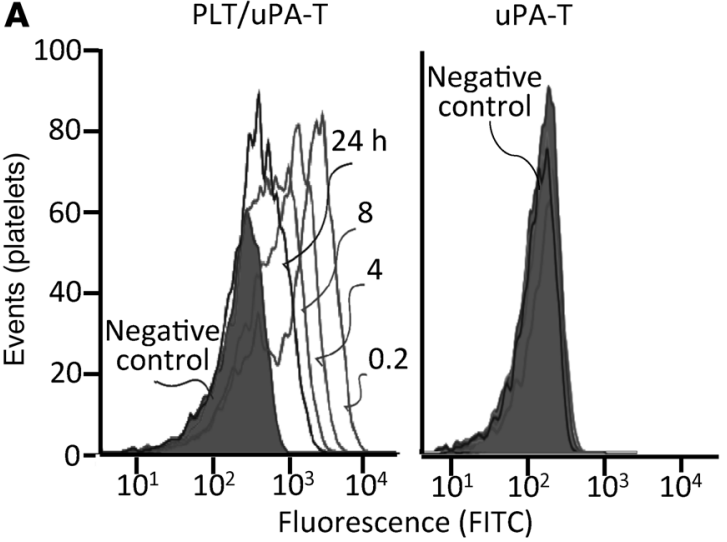

C

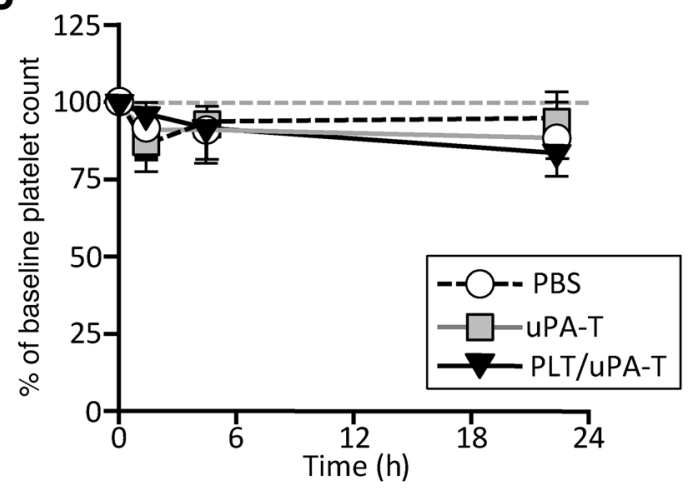

B

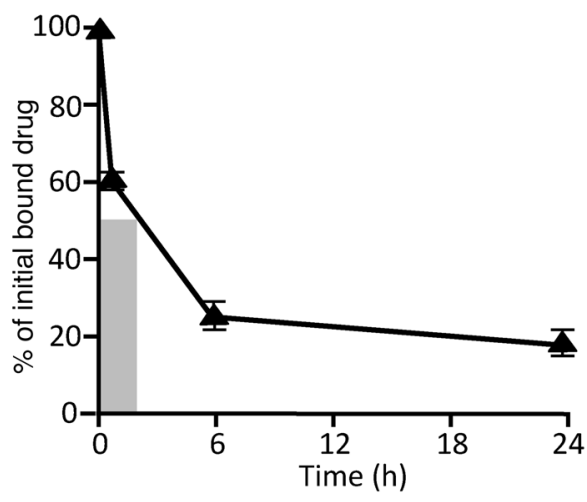

D

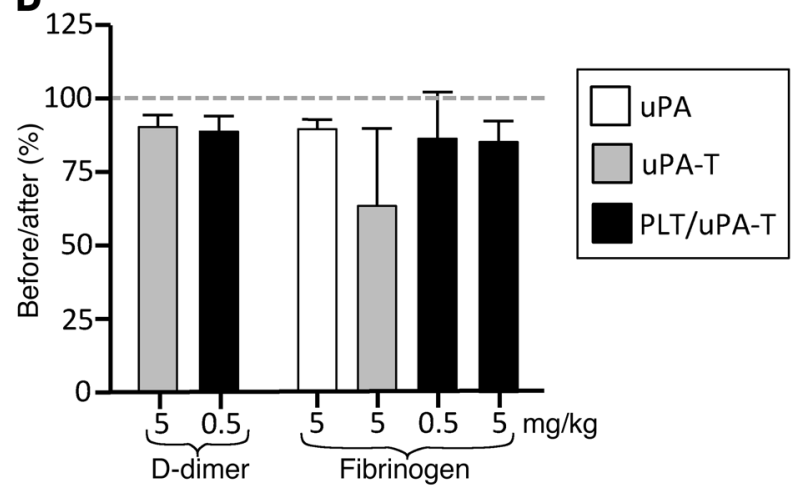

Figure 5. Systemic effects of the uPA-T prodrugs. (A) Representative flow cytometric study of a single-dose infusion of PLT/uPA-T (0.25 mg/kg) and uPA-T $\left(5 \mathrm{mg} / \mathrm{kg}\right.$ ) into hall $b^{+}$mice, followed by measurements of platelet-bound PLT/uPA-T (left) and uPA-T (right). (B) Data represent the mean \pm 1 SEM for 5 studies of infusion of PLT/uPA-T into hallb+ mice and were analyzed as in A for PLT/uPA-T. Gray bar indicates a half-life of $\sim 2$ hours. (C) Effects of infusions of PBS (white circles), UPA-T ( $5 \mathrm{mg} / \mathrm{kg}$, gray squares), or PLT/uPA-T $(0.25 \mathrm{mg} / \mathrm{kg}$, black triangles) on circulating platelet counts at $0,1,4$, and 24 hours after iv. infusion of the drug. The drop in platelet count observed with each intervention to $90 \%$ of baseline was due, we believe, to repeated blood draws. Gray dashed line represents the baseline platelet count with no intervention. (D) D-dimer and fibrinogen levels after a single-bolus, 30-minute infusion of uPA, UPA-T, or PLT/UPA-T were determined. The amount infused is shown on the $x$ axis in $\mathrm{mg} / \mathrm{kg}$. Values were compared with the levels measured prior to prodrug infusion in each animal. Studies were performed at the end of the infusion. Data represent the mean \pm 1 SEM. Dashed lines in $\mathbf{D}$ represents settings in which pre- and post-drug infusion levels were identical. $n=5$ for all studies. None reached statistical significance when compared with the pre-study levels. Data analysis was done by Student $t$ test for drug-treated mice compared with PBS controls.

to be activated, which should prevent inactivation by PAI-1 in the circulation and limit activity to the site of actively growing thrombi; (b) the drug binds tightly to the surface of nonactivated platelets and has a half-life of several hours in the circulation; and (c) PLT/uPA-T proteolytic activity can be blocked using TEA in the event of bleeding. In several murine hemostasis/thrombosis models, PLT/uPA-T inhibited occlusive thrombi from forming after injury, while sparing preexisting thrombi. The agent is suitable for the study of more clinically relevant thrombosis models in baboons. Further studies should provide insight into the role of platelets in clot remodeling and help to develop unique platelettargeting strategies for different clinical needs.

\section{Methods}

\section{Murine lines studied}

The h $\alpha \mathrm{IIb}^{+}$mice used in these studies were transgenic for human integrin $\alpha \mathrm{IIb}(\operatorname{Tg}(\operatorname{ITGA2B}))$. These $\mathrm{h} \alpha \mathrm{IIb}^{+}$mice were backcrossed onto a C57BL/ 6 background more than 10 times, had platelets that expressed haIIb levels at $1 \%$ to $20 \%$ of levels seen on human platelets (15), and were studied concurrently with their WT sibling littermates. These mice were genotyped by PCR analysis of tail genomic DNA using previously described primer pairs and reaction conditions. NOD/SCID/IL-2R $\gamma$-deficient (NSG) mice (23) were produced in pathogen-free animal facilities at the CHOP using breeders obtained from The Jackson Laboratory. Six- to twelve-week-old NSG male mice were studied.

\section{Anti- $\alpha$ llb $\beta 3$ mAbs, generation of scFv chains, and final chimeric prodrugs}

Various antiplatelet mAbs were analyzed by flow cytometry to characterize their binding specificity to human and WT and haIIb ${ }^{+}$mouse platelets (24). The mAb 312.8 was used to produce a platelet-specific $\mathrm{scFv}$. Construction of the $\mathrm{scFv}$ and chimeric prodrug was performed following previously described techniques with modifications (Figure 1A and refs. 25, 26). Total RNA was isolated from $312.8 \mathrm{mAb}$ hybridoma cells and reverse transcribed with RNeasy (QIAGEN) using SMART technology (Clontech) with primers designed to isolate DNA encoding the variable heavy $\left(\mathrm{V}_{\mathrm{H}}\right)$ and variable light $\left(\mathrm{V}_{\mathrm{L}}\right)$ chain domains from mouse hybridoma cells (26). The $\mathrm{V}_{\mathrm{H}}$ and $\mathrm{V}_{\mathrm{L}}$ frag- 
A Nascent: $\mathrm{FeCl}_{3}$ thrombus prevention
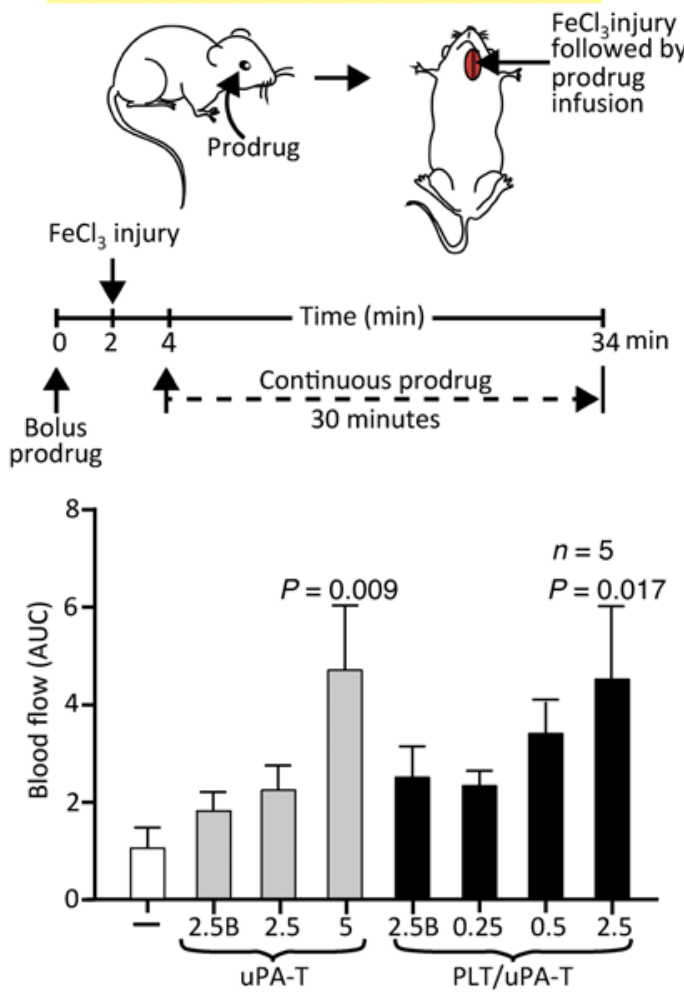

C
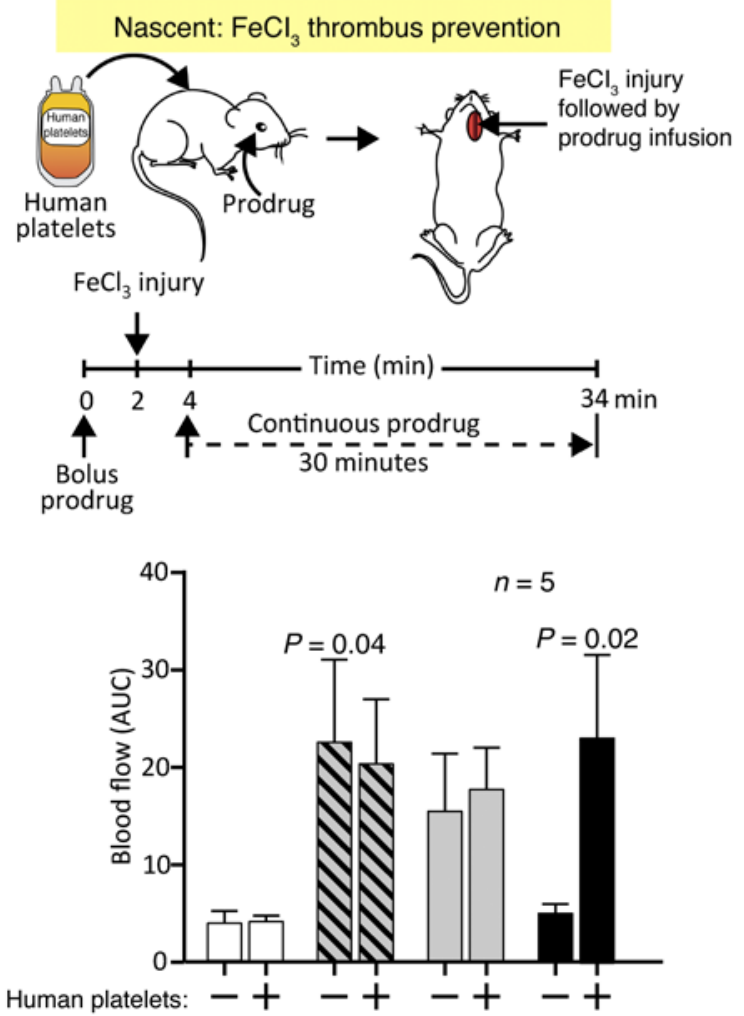

B

Preexisting: tail bleeding

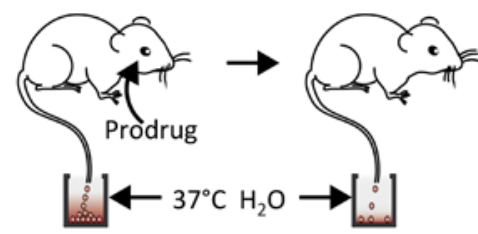

Blood loss vs. Blood loss

(10 min) VS. (30 min)
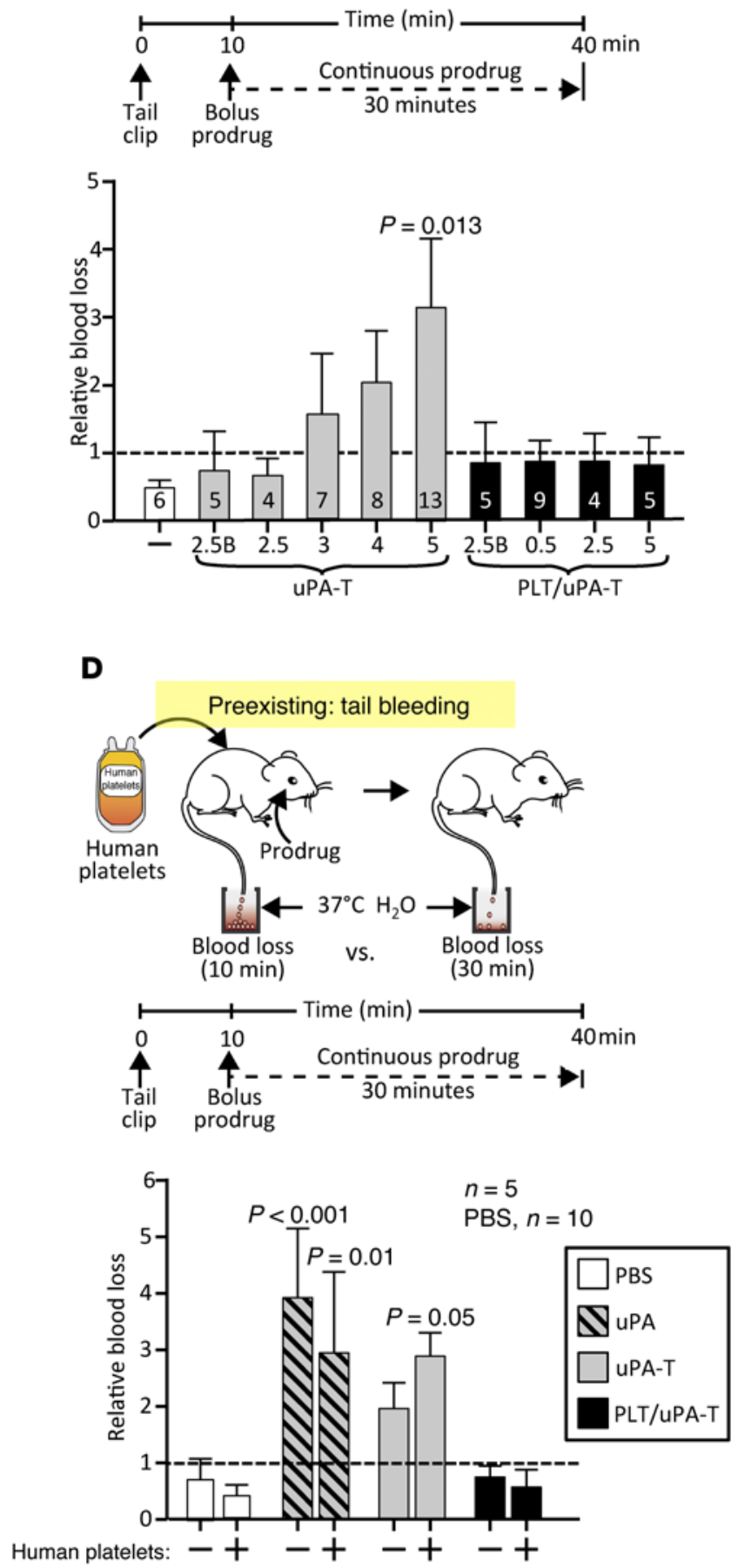
Figure 6. Prodrug efficacy versus nascent thrombi induced by $\mathrm{FeCl}_{3}$-sclerosing versus preexisting thrombi induced by tail-clip injury. (A-D) Schematics of the study and results. Results and schematics shown in $\mathbf{A}$ and $\mathbf{B}$ involve hallb mice. Prodrugs were given as a 2-minute bolus, followed by a 30 -minute infusion dose at the indicated $\mathrm{mg} / \mathrm{kg}$ dose, except for panel $\mathbf{B}$, which refers to a bolus only. Data represent the mean $\pm 1 \mathrm{SEM}$ and are shown by $n$ values. ANOVA multiparametric analysis was performed, and $P$ values are relative to the PBS control. (A) AUC of blood flow in the $\mathrm{FeCl}_{3}$ injury model while receiving the indicated prodrug. AUC for blood flow is shown because, while all of the mice exposed to PBS or bolus uPA-T had total occlusions, none of the other mice developed total occlusions, but some developed partial occlusions that were notable by measuring the AUC. In B, tail-clip blood loss that occurred after the prodrug's infusion was compared with blood loss prior to prodrug infusion. The time to stop bleeding, determined visually during the pre-drug window, was $2.1 \pm 0.4$ minutes, similar to that seen by others in similar models (32). Dashed lines in $\mathbf{A}-\mathbf{D}$ indicate equal blood loss before and after drug exposure. Data shown in $\mathbf{C}$ and $\mathbf{D}$ represent studies analogous to those in $\mathbf{A}$ and $\mathbf{B}$, respectively, but were conducted in NSG mice either infused (+) or not infused (-) with human platelets. The uPA and uPA-T dose for both the bolus and infusion was $5 \mathrm{mg} / \mathrm{kg}$ and $0.5 \mathrm{mg} / \mathrm{kg}$ for PLT/UPA-T. Data shown represent the mean \pm 1 SEM. ANOVA multiparametric analysis was performed, and $P$ values are relative to the PBS control.

ments were cloned into TOPO vectors (Invitrogen) and sequences confirmed (Figure 1D). The $\mathrm{V}_{\mathrm{H}}$ fragment was modified by PCR using a sense primer that introduced the restriction site NcoI (underlined) near the $5^{\prime}$ end (5'-TGCATGTGCATGCCATGGGAGGTGAAGCTGGTGGAGTCAGGGGGAGG-3') and an antisense primer that introduced an 18-amino acid linker (SSGGGGSGGGGGGSSRSS) on the $3^{\prime}$ end to join with the $\mathrm{V}_{\mathrm{L}}$ domain (5'-TTGAGTGAGCACATTTTCGGAAGATCTAGAGGAACCACCCCCACCACCGCCCGAGCCACCGCCACCAGAGGAGGGTGTCGTTTTGGCTGA-3'). The $\mathrm{V}_{\mathrm{L}}$ domain was amplified with a sense primer that complemented part of the $\mathrm{V}_{\mathrm{H}}$ domain (5'-CTCCACCAGCTTCACCTCGGAAGATCTAGAGGAACCACCCCCACCACCGCCCGAGCCACCGCCACCAGAGGAAGTTGGTGCAGCATCAGC-3') and an antisense primer that introduced the amino acid linker (SSSSG) ${ }_{2}$ and a NotI restriction site (underlined) for cloning purposes (5'-AATGCGGCCGCGCCGGAAGAGCTACTACCCGATGAGGAAGAAGTTGGTGCAGCATCAGCCC-3'). Joining of the $\mathrm{V}_{\mathrm{H}}$ and $\mathrm{V}_{\mathrm{L}}$ fragments to form the $s c F v$ was accomplished by PCR DNA amplification using the $\mathrm{V}_{\mathrm{H}}$ sense and the $\mathrm{V}_{\mathrm{L}}$ antisense primers, then digesting with the restriction enzymes NcoI and NotI. The digested construct was cloned into a previously made mouse rbc/uPA-T (8) insect expression vector, resulting in a PLT/uPA-T construct with the UPA-T downstream of the NotI site and a triple FLAG tag on the C-terminus for purification purposes (Figure 1C). Successful cloning was confirmed by restriction analysis of the plasmid and by automated DNA sequencing (not shown). Stable Drosophila cell lines expressing uPA-T or PLT/uPA-T were used to produce recombinant proteins. These proteins were further purified from cell media by affinity chromatography using an M2 (anti-FLAG) affinity column (Sigma-Aldrich), followed by gel filtration chromatography on a Sephacryl SHR100 column (Amersham, GE Healthcare Life Sciences) to 95\% or greater purity, confirmed by SDS-PAGE (data not shown), with a yield of 3 to $5 \mathrm{mg} / \mathrm{l}$ medium. The fusion proteins were concentrated in physiological buffered saline (PBS) $\left(\mathrm{Ca}^{2+}\right.$ - and $\mathrm{Mg}^{2+}$-free; $\left.\mathrm{Gibco}\right)$ to $2 \mathrm{mg}$ / $\mathrm{ml}$, separated into aliquots and stored at $-80^{\circ} \mathrm{C}$.

\section{Murine and human blood samples, platelet preparation, platelet aggregation, and flow cytometric studies}

Human blood was collected in $0.129 \mathrm{M}$ sodium citrate (10:1, vol/vol). Murine whole blood was isolated from the inferior vena cava under direct observation and collected into acid citrate dextrose (ACD) as previously described (15). Platelet-rich plasma (PRP) was separated after centrifugation $(200 \mathrm{~g}, 10$ minutes) at room temperature (RT). The platelets were then isolated from PRP, and prostaglandin E1 (Sigma-Aldrich) was added to a final concentration of $1 \mu \mathrm{M}$. Platelets were then pelleted by centrifugation ( $800 \mathrm{~g}, 10$ minutes) at RT. The pellet was washed in calcium-free Tyrode's buffer (134 mM NaCl, 3 $\mathrm{mM} \mathrm{KCl}, 0.3 \mathrm{mM} \mathrm{NaH}_{2} \mathrm{PO}_{4}, 2 \mathrm{mM} \mathrm{MgCl}, 5 \mathrm{mM}$ HEPES, $5 \mathrm{mM}$ glucose, $0.1 \% \mathrm{NaHCO}_{3}$, and $1 \mathrm{mM}$ EGTA, pH 6.5), and resuspended in CATCH buffer (PBS and 1.5\% bovine serum albumin, $1 \mathrm{mM}$ adenosine, $2 \mathrm{mM}$ theophylline, $0.38 \%$ sodium citrate, all from SigmaAldrich). Platelet counts were determined using a HemaVet counter (Triad Associates). Platelet aggregation studies with the mAb 312.8 were performed as previously described (27) using human PRP adjusted to $3 \times 10^{5}$ platelets/ $\mu$ l with platelet-poor plasma (PPP). Samples of these platelets were either untreated or incubated for 5 minutes at $37^{\circ} \mathrm{C}$ with $20 \mu \mathrm{g} / \mathrm{ml} \mathrm{mAb}$. Platelet aggregation was induced by adding $10 \mu \mathrm{M}$ ADP to the platelet preparations, and light transmission was measured over time in a Kowa AG-10E aggregometer under continuous stirring. Flow cytometry (using a BD FACScan) was used to measure the binding of $1 \mu \mathrm{g} 312.8 \mathrm{mAb}$ and $\mathrm{uPA}-\mathrm{T}$ or PLT/uPA-T to resting or PMA-activated $(50 \mu \mathrm{M})$ washed platelets $\left(1 \times 10^{7} / \mu \mathrm{l}\right)$. To detect platelet-bound uPA-T or PLT/uPA-T, anti-FLAG M2-FITC $\mathrm{mAb}$ (Sigma-Aldrich) and a phycoerythrin-labeled (PE-labeled) anti-human CD41 mAb (eBioscience) were incubated sequentially with the samples for 30 minutes each prior to analysis. To detect bound $312.8 \mathrm{mAb}$, a goat anti-mouse IgG-FITC Ab (eBioscience) was used. In some studies, binding was detected by directly labeling the $312.8 \mathrm{mAb}$ and the fusion protein with Alexa Fluor 647 (Invitrogen) and incubating $1 \mu \mathrm{g}$ labeled protein with platelets before analysis.

\section{PLT/uPA-T-binding specificity and affinity studies}

Binding specificity of PLT/uPA-T was analyzed by flow cytometry using human and murine $\mathrm{WT}$ and $\mathrm{h} \alpha \mathrm{IIb}^{+}$platelets. Binding was also assessed by radiolabeling the PLT/uPA-T with ${ }^{125} \mathrm{I}-\mathrm{Na}$ (PerkinElmer) in iodination tubes (Thermo Scientific). After blocking with 5\% PBS/ BSA and washing with PBS/Tween-20, various concentrations of PLT/ uPA-T $(100 \mu \mathrm{l} /$ well $)$ were incubated with human $\alpha \mathrm{IIb} \beta 3$ or BSA coated wells for 1 hour at $37^{\circ} \mathrm{C}$. Nonbound material was removed by washing with PBS/Tween-20, and residual radioactivity was measured on a $\gamma$-counter (PerkinElmer).

\section{PLT/UPA-T thrombin activation and specificity of activation}

Activation of PLT/uPA-T by thrombin was measured using bovine fibrinogen (Sigma-Aldrich) dissolved in PBS to a final concentration of $3 \mathrm{mg} / \mathrm{ml}$. Clotting was induced by addition of $20 \mathrm{mM} \mathrm{CaCl}_{2}$ and 0.2 units $/ \mathrm{ml}$ thrombin (Sigma-Aldrich), and the resulting solution was immediately added to a 24 -well cell culture plate $(0.75 \mathrm{ml} /$ well), where clots were allowed to mature for 30 minutes. UPA-T or PLT/ uPA-T was activated by incubation with $20 \mathrm{nM}$ bovine thrombin 


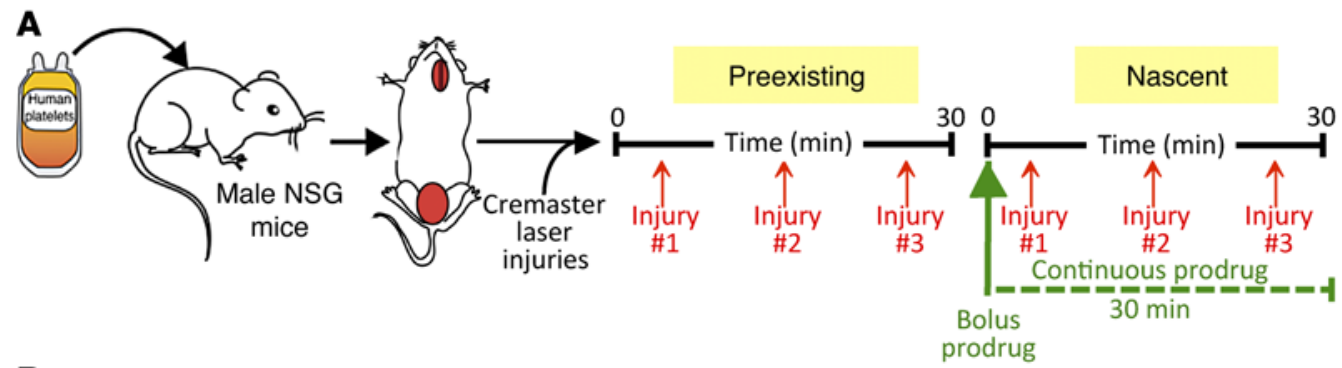

B
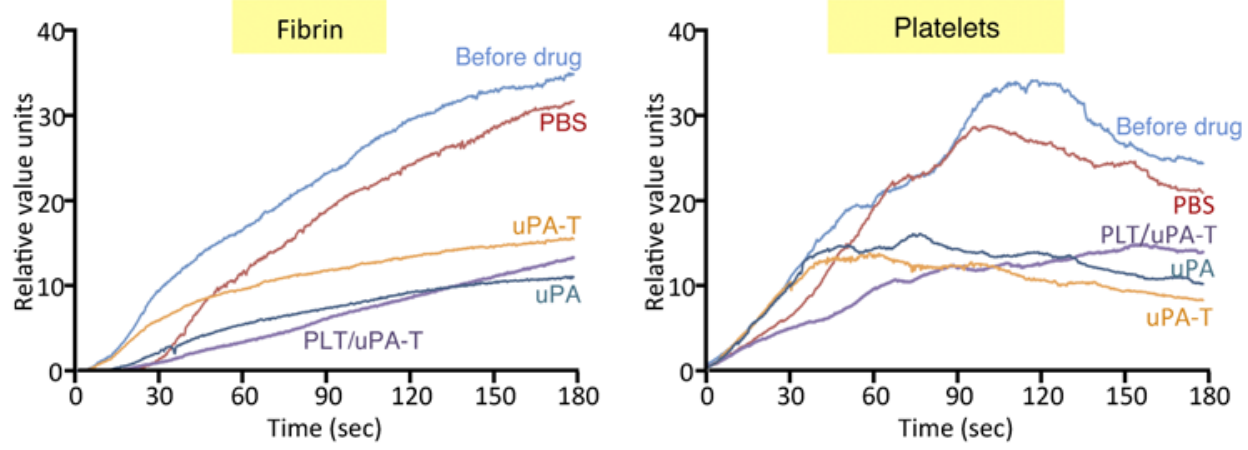

C
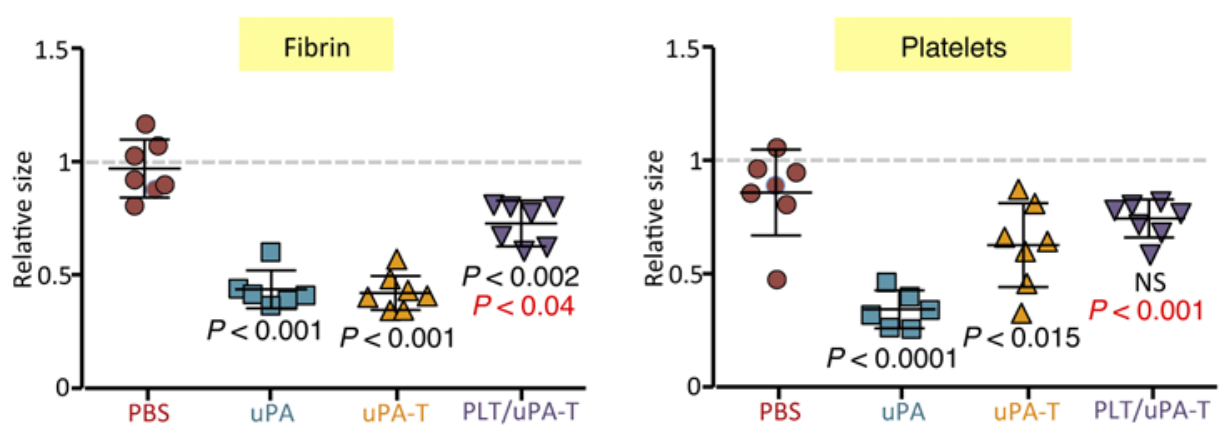

Figure 7. Prodrug efficacy in nascent versus preexisting thrombi in the cremaster laser injury model. Xenotransfused NSG mice were used for these studies, as for Figure 6, C and D. (A) Schematic of studies. The degree of prevention of growth of nascent thrombi under the bolus infusion of a prodrug is an indication of the thromboprophylactic effectiveness of the tested prodrug. Laser injuries performed prior to drug bolus infusions generated the preexisting thrombi. The degree by which they decreased in size after a bolus infusion of a prodrug was an indication of the effect of the tested prodrug on lysing preexisting thrombi. (B) Platelet and fibrin accumulation in nascent injuries during bolus infusion of PBS, $5 \mathrm{mg} / 5 \mathrm{mg}$ uPA or uPA-T, or $0.125 \mathrm{mg} / 0.125$ mg PLT/UPA-T. $n=5$ animals per arm, with up to 5 pre- and post-drug injuries per animal. (C) Relative size of the thrombus as indicated by measuring accumulated platelets and fibrin in preexisting injuries that had then been exposed to a bolus infusion of the same drugs as used in $\mathbf{B}$. Size is shown relative to the preexisting size, i.e., prior to drug exposure. ANOVA multiparametric analysis was performed, and $P$ values are relative to the PBS control. $P$ values shown in black are relative to the PBS control, and values in red are relative to uPA.

(Amersham, GE Healthcare Life Sciences) for 1 hour. Serial dilutions $(50 \mu \mathrm{l} /$ well $)$ of the activated prodrugs were added on top of each clot at $37^{\circ} \mathrm{C}$ for 12 hours, and zones of lysis were measured (8). This fusion protein and parental uPA-T have similar specific fibrinolytic activity. Samples were supplemented with $50 \mathrm{nM}$ TEA to test the fusion protein's efficacy at preventing proteolysis.

The kinetics of PLT/uPA-T and uPA-T activation by thrombin was studied over a 1.5-hour period using the uPA-specific chromogenic peptide substrate pyroGlu-Gly-Arg-pNA-HCl (Spectrozyme uPA; American Diagnostica Inc.). PLT/uPA-T or uPA-T (10 nM final concentration) was mixed with Spectrozyme uPA (1.67 mM final concentration) in the absence or presence of $1 \mathrm{nM}$ bovine thrombin (Amersham, GE Healthcare Life Sciences). Thrombin, PLT/uPA-T, and $\mathrm{UPA}-\mathrm{T}$ were each used alone as negative controls to ensure that they did not possess amidolytic activity toward the Spectrozyme
uPA substrate. Cleavage of the chromogenic substrate by activated PLT/uPA-T or UPA-T was monitored by measuring the optical density at $405 \mathrm{~nm}$ on an ELISA plate reader every 15 seconds. To study the sensitivity of the activation reaction to antithrombin III, thrombin was premixed with Antithrombin III (Sigma-Aldrich) (thrombin/antithrombin ratio of 1:2) and then added to the mixture of PLT/uPA-T or UPA-T with Spectrozyme uPA substrate. The kinetics of activation of PLT/uPA-T or UPA-T was measured as above.

To affirm thrombin specificity, PLT/uPA-T-generated amidolytic activity was tested after a 1-hour incubation with $5 \mathrm{nM}$ thrombin at $37^{\circ} \mathrm{C}$. These studies were repeated with human elastase, chymase, tryptase, plasmin, heat-inactivated thrombin, activated protein C, or kallikrein (all from Sigma-Aldrich). Thrombin was inactivated by heating at $100^{\circ} \mathrm{C}$ for 30 minutes to distinguish between the requirement for its catalytic and noncatalytic activi- 
ties. The resulting amidolytic activity was assayed in activity buffer (50 mM Tris, pH 7.5, $30 \mathrm{KIU} / \mathrm{ml}$ aprotinin, and $33 \mathrm{U} / \mathrm{ml}$ hirudin) as described previously (28).

\section{Prodrug half-life and activity in hall $b^{+}$mice}

uPA-T $(5 \mathrm{mg} / \mathrm{kg})$, PLT/uPA-T $(0.25 \mathrm{mg} / \mathrm{kg})$, or PBS was labeled with Alexa Fluor 647 (Invitrogen, Thermo Fisher Scientific) and infused i.v. into haIIb mice. The prodrug doses were chosen on the basis of their efficaciousness in the microfluidic system (Figure 5) and/or in vivo (Figures 6 and 7). Retroorbital blood was collected into a minicapillary blood collection tube (RAM Scientific) prior to infusions and at time points extending up to 72 hours after infusion. Platelet-bound PLT/ uPA-T and uPA-T were analyzed by flow cytometry. Platelet counts were measured at time points after a single infusion for up to 24 hours using a HemaVet counter (Triad Associates). Fibrinogen and D-dimer levels were measured in mice before and after prodrug infusions using the Mouse Fibrinogen ELISA kit (Molecular Innovations) and a Mouse FDP ELISA kit (Novatein Biosciences), respectively.

\section{Microfluidic studies of thrombolysis}

Human PRP was isolated from whole blood collected in $3.8 \%$ sodium citrate $(1: 10, \mathrm{v} / \mathrm{v})$. uPA-T or PLT/uPA-T $(10 \mu \mathrm{g} / \mathrm{ml}$ each) and calcein AM $(2 \mu \mathrm{M})$ (Invitrogen) were added for 30 minutes at RT. The PRP was then passed through a column packed with $20 \mathrm{ml}$ Sepharose CL-2B (GE Healthcare), then prewashed and equilibrated with calcium-free Tyrode's buffer. Analysis of platelet-bound drug was performed by flow cytometry. These gel-filtered platelets were added back to the PRP-depleted blood to reconstitute "whole blood." The blood was then activated by either adding recombinant FVIIa (80 $\mathrm{nM}$, ref. 16) or inducing an HIT-like prothrombotic state by adding $10 \mu \mathrm{g} / \mathrm{ml}$ platelet factor 4 (PF4) and the HIT-like mAb KKO $(50 \mu \mathrm{g} / \mathrm{ml})$ as previously described (17) plus Alexa Fluor 647-labeled fibrinogen (Invitrogen). Samples were partially recalcified by adding $5 \mathrm{mM} \mathrm{CaCl}_{2}$. The samples were then flowed through a BioFlux microchannel (Fluxion Biosciences) coated with $20 \mu \mathrm{g} / \mathrm{ml}$ human $v W F$ (American Diagnostica) for 20 minutes at 20 dyne $/ \mathrm{cm}^{2}$. Image and video acquisition was obtained with an Observer Z.1 inverted microscope (Zeiss). The surface area covered by calcein-labeled platelets and Alexa Fluor 647-labeled fibrinogen/fibrin was analyzed using BioFlux Montage software (Fluxion Biosciences).

\section{Murine hemostasis models to demonstrate PLT/UPA-T thromboprophylaxis}

$\mathrm{FeCl}_{3}$ carotid artery and tail-clipping injury murine models. Two models of hemostasis/thrombosis were used to evaluate whether the PLT/uPA-T targeted nascent thrombi, while sparing preexisting ones. In the first model, nascent carotid arterial thrombi were induced in haIIb $b^{+}$mice using $\mathrm{FeCl}_{3}$ (11), and preexisting thrombi were studied using a tail-clipping injury model $(29,30)$. The models were modified to also study NSG, rather than haIIb ${ }^{+}$, mice infused with human platelets. In both iterations, mice were first anesthetized by i.p. injection of pentobarbital $(80 \mathrm{mg} / \mathrm{kg}$ nembutal; Ovation Pharmaceuticals). NSG mice were then infused retro-orbitally with washed human platelets so that approximately $20 \%$ of the circulating platelets were of human origin (determined by flow cytometry using a combination of PE-conjugated rat anti-mouse CD41 and FITC-conjugated mouse anti-human CD41a). For the carotid artery injury study, $12.5 \%-15 \% \mathrm{FeCl}_{3}$ was applied for 2 minutes (11). An i.v. bolus of the test prodrug was administered 2-2.5 minutes before injury as a bolus alone or with a subsequent continuous infusion for 30 minutes. Total flow and time to the first complete occlusion of more than 7 minutes were recorded. Preexisting tail-injury thrombi were produced by amputating an 8-mm terminal tail segment, which was then placed in a collection tube containing sterile water at $37^{\circ} \mathrm{C}$ for 10 minutes. The tail was then placed in a new collection tube, and a bolus of LMW uPA or its derivative UPA-T or PLT/ uPA-T was administered i.v., followed by continuous infusion for 30 minutes. The amount of hemoglobin in the water was measured with a spectrophotometer as described previously $(31,32)$. Briefly, the hemolyzed whole blood and water mixture was centrifuged at $15,000 \mathrm{~g}$ for 5 minutes. Aliquots $(10 \mu \mathrm{l})$ of clarified, stroma-free supernatant were diluted 10-fold in a 96-well microplate (Corning), and light absorbance was measured at $576 \mathrm{~nm}$ with a SpectraMax 190 Microplate Reader (Molecular Devices). Blood loss during the 30-minute time window was normalized for the blood loss during the earlier 10-minute observation period to account for differences in the extent of initial injuries and severity of initial bleeding.

Early and late cremaster arteriole laser injury murine model. To confirm the potential of PLT/uPA-T to lyse nascent thrombi while sparing preexisting ones, we used the cremaster laser-induced thrombosis system (33) as a second model. NSG male mice were studied after being anesthetized using i.p. injected sodium pentobarbital $(60 \mathrm{mg} / \mathrm{kg})$. The mice were maintained under anesthesia with the same anesthetic delivered via a catheterized jugular vein at $5 \mathrm{mg} / \mathrm{ml}$ throughout the experiment. Human platelets were resuspended in PBS and infused into the jugular vein, followed by Alexa Fluor 647- or 488-conjugated rat anti-mouse CD41 Fab fragments (BD Biosciences) to label endogenous mouse platelets and Alexa Fluor 647-labeled anti-fibrin 59D8 Ab (34). Laser injuries were induced in cremaster arterioles before and after PBS or uPA or UPA-T or PLT/uPA-T by i.v. bolus plus continuous infusion. Wide-field images of thrombi were captured using SlideBook 6.0 (Intelligent Imaging Innovations), and intensity of signal over the thrombus was used to measure incorporated platelets and fibrin (33). Locations of predrug injuries were marked in the software to revisit and recapture after the bolus and continuous infusion of drug or PBS. Analysis of images was performed using SlideBook 6.0 (35). Data were collected over a 3-minute period for each injury. Up to 8 injuries were made in each mouse.

\section{Statistics}

Data in Figures 4 and 5 were compared with WT controls by Student's $t$ test using Prism 5.0 (GraphPad Software). Data in Figures 6 and 7 were analyzed using ANOVA with post-test comparisons against controls or within groups by Dunnett's correction where appropriate. Multiplicity -adjusted $P$ values are reported. For all analyses, a $P$ value of less than 0.05 was considered significant.

\section{Study approval}

Human blood samples were obtained from healthy volunteers, who provided written informed consent. Studies were approved by the IRB of the CHOP. All animal experiments were approved by the IACUC of the CHOP, and all investigators adhered to NIH guidelines for the care and use of laboratory animals. 


\section{Author contributions}

REF and SZ developed the construct, expressed and purified the proteins, conducted the initial in vitro studies, and contributed to the intellectual development of the project and the preparation of the manuscript. HSA completed the in vitro studies. HSA and VH carried out the in vivo studies and assisted with the preparation of the manuscript. MAK and YW assisted with the platelet-related studies. MPL performed the statistical analysis of the results. DLS helped with the design and data interpretation related to the scFv. DWB and RHA contributed the Abs screened and to the design and interpretation of related studies. DDM assisted with the baboon studies and their interpretation. VS assisted with developing the ScFV construct and with the pharmacokinetic studies. DBC, VRM, and MP designed the project, oversaw its performance, provided data interpretation, and assisted with manuscript preparation.

\section{Acknowledgments}

This work was supported by NIH grants PO1 HL40387 (to D.B. Cines, V.R. Muzykantov, M. Poncz, and S. Zaitsev), R01 HL013629 (to D.W. Bougie and R.H. Aster), and T32 HL07971 (to R.E. Fuentes).

Address correspondence to: Mortimer Poncz, The Children's Hospital of Philadelphia, 3615 Civic Center Blvd., ARC, Rm. 317, Philadelphia, Pennsylvania 19104, USA. Phone: 215.590.4374; E-mail: poncz@email.chop.edu.
1. Geerts WH, et al. Prevention of venous thromboembolism: the Seventh ACCP Conference on Antithrombotic and Thrombolytic Therapy. Chest. 2004;12(3 suppl):338S-400.

2. Stein PD, Kayali F, Olson RE. Estimated case fatality rate of pulmonary embolism, 1979 to 1998. Am J Cardiol. 2004;93(9):1197-1199.

3. Bell WR Jr. Evaluation of thrombolytic agents. Drugs. 1997;54(suppl 3):11-16.

4. Armstead WM, Cines DB, Higazi AA. Plasminogen activators contribute to impairment of hypercapnic and hypotensive cerebrovasodilation after cerebral hypoxia/ischemia in the newborn pig. Stroke. 2005;36(10):2265-2269.

5. Armstead WM, Cines DB, Bdeir KH, Bdeir Y, Stein SC, Higazi AA. uPA modulates the age-dependent effect of brain injury on cerebral hemodynamics through LRP and ERK MAPK. J Cereb Blood Flow Metab. 2009;29(3):524-533.

6. Simpson D, Siddiqui MA, Scott LJ, Hilleman DE. Reteplase: a review of its use in the management of thrombotic occlusive disorders. Am J Cardiovasc Drugs. 2006;6(4):265-285.

7. Agnelli G, Becattini C. Acute pulmonary embolism. N Engl JMed. 2010;363(3):266-274.

8. Zaitsev S, et al. Sustained thromboprophylaxis mediated by an RBC-targeted pro-urokinase zymogen activated at the site of clot formation. Blood. 2010;115(25):5241-5248.

9. Kahr WH, et al. Platelets from patients with the Quebec platelet disorder contain and secrete abnormal amounts of urokinase-type plasminogen activator. Blood. 2001;98(2):257-265.

10. Blavignac J, Bunimov N, Rivard GE, Hayward CP. Quebec platelet disorder: update on pathogenesis, diagnosis, and treatment. Semin Thromb Hemost. 2011;37 (6):713-720.

11. Kufrin D, et al. Antithrombotic thrombocytes: ectopic expression of urokinase-type plasminogen activator in platelets. Blood. 2003;102(3):926-933.

12. Erickson LA, Ginsberg MH, Loskutoff DJ. Detection and partial characterization of an inhibitor of plasminogen activator in human platelets. JClin Invest. $1984 ; 74(4): 1465-1472$.

13. Wagner CL, Mascelli MA, Neblock DS, Weisman HF, Coller BS, Jordan RE. Analysis of GPIIb/IIIa receptor number by quantification of $7 \mathrm{E} 3$ binding to human platelets. Blood. 1996;88 (3):907-914.
14. Christensen U. Allosteric effects of some antifibrinolytic amino acids on the catalytic activity of human plasmin. Biochim Biophys Acta. 1978;526(1):194-201.

15. Thornton MA, Zhang C, Kowalska MA, Poncz $M$. Identification of distal regulatory regions in the human alpha IIb gene locus necessary for consistent, high-level megakaryocyte expression. Blood. 2002;100(10):3588-3596.

16. Margaritis P, Arruda VR, Aljamali M, Camire RM, Schlachterman A, High KA. Novel therapeutic approach for hemophilia using gene delivery of an engineered secreted activated Factor VII. J Clin Invest. 2004;113(7):1025-1031.

17. Arepally GM, et al. Characterization of a murine monoclonal antibody that mimics heparininduced thrombocytopenia antibodies. Blood. 2000;95(5):1533-1540.

18. Stalker TJ, et al. A systems approach to hemostasis: 3. Thrombus consolidation regulates intrathrombus solute transport and local thrombin activity. Blood. 2014;124(11):1824-1831.

19. Bode C, et al. Platelet-targeted fibrinolysis enhances clot lysis and inhibits platelet aggregation. Circulation. 1991;84(2):805-813.

20. Köhler M, et al. Half-life of single-chain urokinasetype plasminogen activator (scu-PA) and twochain urokinase-type plasminogen activator (tcu-PA) in patients with acute myocardial infarction. Thromb Res. 1991;62(1-2):75-81.

21. Hohmann JD, et al. Delayed targeting of CD39 to activated platelet GPIIb/IIIa via a singlechain antibody: breaking the link between antithrombotic potency and bleeding? Blood. 2013;121(16):3067-3075.

22. Wang $X$, et al. Towards effective safe thrombolysis thromboprophylaxis: preclinical testing of a novel antibody-targeted recombinant plasminogen activator directed against activated platelets. Circ Res. 2014;114(7):1083-1093.

23. Ito M, et al. NOD/SCID/gamma(c)(null) mouse: an excellent recipient mouse model for engraftment of human cells. Blood. 2002;100(9):3175-3182.

24. Bougie DW, Birenbaum J, Rasmussen M, Poncz M, Aster RH. Quinine-dependent, plateletreactive monoclonals mimic antibodies found in patients with quinine-induced immune thrombocytopenia. Blood. 2006;113(5):1105-1111.
25. Ding BS, et al. Prophylactic thrombolysis by thrombin-activated latent prourokinase targeted to PECAM-1 in the pulmonary vasculature. Blood. 2008;111(4):1999-2006.

26. Dübel S, et al. Isolation of IgG antibody Fv-DNA from various mouse and rat hybridoma cell lines using the polymerase chain reaction with a simple set of primers. J Immunol Methods. 1994;175(1):89-95.

27. Rauova L, et al. Monocyte-bound PF 4 in the pathogenesis of heparin-induced thrombocytopenia. Blood. 2010;116(23):5021-5031.

28. Ding BS, et al. Endothelial targeting of a recombinant construct fusing a PECAM-1 single-chain variable antibody fragment $(\mathrm{scFv})$ with prourokinase facilitates prophylactic thrombolysis in the pulmonary vasculature. Blood. 2005;106(13):4191-4198.

29. Yarovoi HV, et al. Factor VIII ectopically expressed in platelets: efficacy in hemophilia A treatment. Blood. 2003;102(12):4006-4013.

30. Greene TK, Schiviz A, Hoellriegl W, Poncz M, Muchitsch EM. Animal Models Subcommittee of the Scientific Standardization Committee of the ISTH. Towards a standardization of the murine tail bleeding model. J Thromb Haemost. 2010;8(12):2820-2822.

31. Ray GB, Blair HA, Thomas CI. The spectrophotometric determination of certain blood pigments. J Biol Chem. 1932;98:63-83.

32. Liu Y, Jennings NL, Dart AM, Du XJ. Standardizing a simpler, more sensitive and accurate tail bleeding assay in mice. World J Exp Med. 2012;2(2):30-36.

33. Neyman M, Gewirtz J, Poncz M. Analysis of the spatial and temporal characteristics of platelet-delivered factor VIII-based clots. Blood. 2008;112(4):1101-1108.

34. Courtney MA, Bunce LA, Neroni LA, Simpson-Haidaris PJ. Cloning of the complete coding sequence of rat fibrinogen $B$ beta chain cDNA: interspecies conservation of fibrin beta 15-42 primary structure. Blood Coagul Fibrinolysis. 1994;5(4):487-496.

35. Falati S, Gross P, Merrill-Skoloff G, Furie BC, Furie B. Real-time in vivo imaging of platelets, tissue factor and fibrin during arterial thrombus formation in the mouse. Nat Med. 2002;8(10):1175-1181. 